Revue interdisciplinaire d'Humanités

\title{
Esthétique de la politique ou politique de l'esthétique ? Jouer Rancière contre lui-même
}

\author{
Raphaël Jaudon
}

\section{(2) OpenEdition}

1 Journals

Édition électronique

URL : http://journals.openedition.org/essais/812

DOI : 10.4000/essais.812

ISSN : 2276-0970

Éditeur

École doctorale Montaigne Humanités

\section{Édition imprimée}

Date de publication : 15 septembre 2020

Pagination : 15-25

ISBN : 979-10-97024-08-6

ISSN : 2417-4211

\section{Référence électronique}

Raphaël Jaudon, « Esthétique de la politique ou politique de l'esthétique ? Jouer Rancière contre luimême », Essais [En ligne], 16 | 2020, mis en ligne le 28 septembre 2020, consulté le 12 octobre 2020. URL : http://journals.openedition.org/essais/812 ; DOI : https://doi.org/10.4000/essais.812 


\section{Esthétique de la politique ou politique de l'esthétique? Jouer Ranciêre contre lui-même}

\section{Raphaël Jaudon}

Depuis une vingtaine d'années, la pensée de Jacques Rancière s'est imposée comme un passage obligé de la réflexion sur l'art politique, en réactualisant le projet d'une lecture dissensuelle des opérations poétiques. Ce succès s'explique peut-être par la volonté de l'auteur de décloisonner la politique pour en porter les problèmes jusque dans le champ de l'art. Quelle que soit la discipline envisagée, on retrouve sous sa plume un certain nombre de thèmes récurrents : l'égalité, l'émancipation, le peuple ou encore la critique de la pensée hiérarchique. Au premier abord, son système présente donc un haut degré de cohérence, et constitue une tentative remarquable pour renouveler notre conception de l'engagement artistique.

Toutefois, pour qui accepte de la lire en détails, la philosophie de Rancière demeure structurée selon une division des tâches relativement classique : il y a d'un côté les scènes de la politique, de l'autre celles de l'art ; d'un côté l'" esthétique de la politique ", de l'autre la "politique de l'esthétique », pour reprendre ses propres termes. Deux processus que le philosophe tient à maintenir distincts, dans la pensée comme dans la pratique ${ }^{1}$. A partir de ce constat, le présent article aimerait s'interroger sur la nécessité de cette séparation, afin d'évaluer l'écart qui peut exister entre la philosophie politique et l'esthétique de Rancière.

\section{Esthétique de la politique : le versant sensible de l'action}

Forgée au contact du marxisme, la pensée de Rancière en a conservé la dimension globale : en dernière analyse, son objectif est de lutter contre les mécanismes de domination qui structurent la société. La philosophie politique suppose une vue d'ensemble. À partir de cette détermination minimale, l'originalité de Rancière consiste à approcher la totalité sociale par le biais de son esthétique, terme sous lequel il faut entendre la branche de la philosophie qui se préoccupe des apparences et des sensations, et non celle qui délimite le territoire de l'art.

1 Jacques Rancière, Malaise dans l'esthétique, Paris, Galilée, 2004, p. 66. 
Du déplacement qu'il opère à partir du marxisme, Rancière finira par tirer un concept dont la fortune critique n'est plus à démontrer : celui de "partage du sensible ». Il désigne par là une certaine " distribution de la parole, du temps, de l'espace $»^{2}$ qui s'impose aux individus dans une société donnée, en fonction de la place qu'ils y occupent et des activités qu'ils y conduisent ${ }^{3}$. Toute société fonctionne comme si ses membres avaient procédé au départ à une séance de partage fortement inégalitaire, non seulement des richesses et du pouvoir, mais également des chances d'être vus, entendus et pris en compte. Distribution formelle et symbolique, donc, autant que culturelle ou économique : il y a les individus que l'on voit et ceux que l'on ne voit pas, ceux dont la voix porte et ceux qui peinent à y faire entendre un logos, ceux qui ont leur place dans la mémoire collective et ceux dont la présence est tenue pour indésirable ${ }^{4}$. Ce que nous dit Rancière, c'est que la politique d'une cité peut être lue au prisme de son esthétique, donc de la distribution des corps et des visages qu' elle propose au regard de l'observateur.

Le partage du sensible, en tant que modèle, entend décrire l'entrelacement du sensible et de l'intelligible sous l'action de ce domaine d'activité que l'on nomme " politique ", et qui concerne à la fois l'exercice de l'État et les initiatives individuelles. Le concept proposé par Rancière atteste simultanément de deux réalités contradictoires : d'une part, nous habitons tous le même espacetemps; d'autre part, nous n'y voyons et n'y entendons pas tous la même chose. Le partage signale à la fois l'un et le multiple, le commun et la division. Dès lors, tout acte politique est une décision quant à l'apparence de ce monde, aux formes de subjectivité qu'il est possible d'y rencontrer, et aux modes d'expérience disponibles pour ses habitants.

C'est à ce titre que l'on peut parler, pour Rancière, d'une esthétique de la politique. L'expression désigne à la fois le versant sensible de l'activité politique (politicienne, militante ou citoyenne) et le geste de recadrage qui consiste à en faire l'enjeu principal du travail critique et philosophique (" la politique est d'abord une bataille sur les données sensibles $"^{5}$ ). Cette thèse explique les réserves de Rancière à l'égard des auteurs qui envisagent le lien entre esthétique et politique comme une contamination ponctuelle. Par exemple, lorsque Walter Benjamin observe un mouvement d' " esthétisation de la politique » à l'œuvre dans le futurisme et le fascisme, il y voit la menace d'une dépolitisation de la politique par le moyen de l'art : les futuristes font l'éloge de la guerre en feignant de ne pas remarquer que ses canonnades, si admirables soient-elles,

2 Jacques Rancière, «Littérature, politique, esthétique. Aux abords de la mésentente démocratique " (2000), in Et tant pis pour les gens fatigués, Paris, Amsterdam, 2009, p. 155.

3 Jacques Rancière, Le Partage du sensible. Esthétique et politique, Paris, La Fabrique, 2000, p. $12-14$.

4 Jacques Rancière, La Mésentente. Politique et philosophie, Paris, Galilée, 1995, p. 44-45.

5 Jacques Rancière, «Littérature, politique, esthétique », op. cit., p. 159 (je souligne). 
causent des dommages bien réels, et qu'il faut que des hommes aient effectivement perdu la vie pour que soient produites les " odeurs de décomposition " dont ils exaltent la beauté et l'intensité . Chez Benjamin, l'idée d'" esthétisation de la politique " laisse entendre que la situation est exceptionnelle. Il faudrait l'ingéniosité perverse de quelques artistes pour appliquer à la guerre ou au pouvoir le jugement désintéressé qui sied aux choses de l'art. Pour Rancière, en revanche, l'esthétisation est toujours déjà là ${ }^{7}:$ il n'existe pas d'acte politique qui ne se donne en même temps comme un phénomène sensible.

Un tel postulat devrait permettre d'offrir une réponse satisfaisante au problème de la politisation de l'art, auparavant dominé par les théories sartriennes de l'engagement, qui ont pour inconvénient de rabattre la politique de l'œuvre sur celle de l'artiste ${ }^{8}$. En faisant du monde des apparences le terrain privilégié de la lutte politique, la notion de partage du sensible met l'accent sur les formes sans pour autant opposer la composante esthétique de l'art à sa composante idéologique. Il semblerait donc légitime d'y adosser une théorie de l'art politique.

Or, c'est précisément ce que Rancière ne fait pas.

\section{Politique de l'esthétique : les moyens propres de l'art}

Lorsqu'il est question de penser la politisation des œuvres, l'ambition de Rancière est de cerner la part de l'activité politique qui appartiendrait exclusivement aux arts, qu'ils posséderaient en propre. Le philosophe renvoie donc dos à dos les pratiques de l'art et celles de l'action, au motif que les unes et les autres ne sauraient être jugées selon les mêmes critères :

Le fond du problème, c'est qu'il n'y a pas de critère d'adéquation entre politique de l'esthétique et esthétique de la politique. Cela n’a rien à voir avec ce que certains disent, à savoir qu'il ne faut pas mélanger l'art avec la politique. De toute façon, ils se mélangent, de toute façon la politique a son esthétique, et l'esthétique a sa politique. Mais il n'y a pas de formule d'adéquation.'

À première vue, la remarque est prudente. Elle se charge de nuancer les aspirations révolutionnaires des artistes en soulignant l'écart qui peut exister entre leurs intentions et l'efficacité réelle des œuvres, répétant ainsi le principe de précaution énoncé dans Le Spectateur émancipé : avant d'espérer qu'un film ou qu'une pièce de théâtre parvienne à changer le monde, il est important de

6 Walter Benjamin, "L'œuvre d'art à l'époque de sa reproductibilité technique " (dernière version, 1939), trad. Maurice de Gandillac et Rainer Rochlitz, in Euvres III, Paris, Gallimard, 2000, p. 314-316.

7 Jacques Rancière, La Mésentente, op. cit., p. 38-39.

8 Jacques Rancière, «Le coup double de l'art politisé » (2006), in Et tant pis pour les gens fatigués, op. cit., p. 511.

$9 \quad$ Ibid., p. 512-513. 
reconnaitre que « les mots sont seulement des mots et les spectacles seulement des spectacles ${ }^{10}$. La cible de Rancière, c'est d'abord la naïveté de certains créateurs et commentateurs pressés de voir dans l'art le geste politique le plus noble qui soit. Néanmoins, cette prudence n'est pas sans effet sur la théorie. On peut en effet s'interroger sur le fonctionnement de cette "politique de l'esthétique " dont les effets émancipateurs agissent "indépendamment des souhaits que peuvent avoir les artistes de servir telle ou telle cause ${ }^{11}$. Il est vrai que ce sont toujours des œuvres qui sont vues, commentées, jugées, et non des intentions. Mais cela signifie-t-il pour autant que les intentions des artistes ne tiennent pour rien dans la puissance politique de l'art ? C'est bien cette thèse radicale qui est défendue dans Le Spectateur émancipé. Pour Rancière, non seulement la contribution de l'art à l'action politique ne se donne pas sous la forme d'une efficacité " calculable ", mais il est indifférent que l'œuvre obéisse à un quelconque projet de remise en question de l'ordre existant. La politique de l'art "précède les politiques des artistes " ${ }^{12}$.

C'est dire combien cette politique de l'esthétique paraît éloignée de ce que l'on entend traditionnellement sous le terme " politique » - y compris tel qu'il intervient dans l'expression ranciérienne d'" esthétique de la politique ". Quelle est donc la substance de cette politique qui semble si chère aux yeux de Rancière, et pourtant si étrangère au commun des mortels ?

Pour qui connaît les préoccupations récurrentes du philosophe, la réponse ne fait aucun doute : l'égalité. Chez Rancière, l'égalité est le concept politique par excellence, celui à l'aune duquel toute action, toute pensée, toute œuvre peut être mesurée. À condition bien sûr de la définir de la manière la plus radicale qui soit : «l'égalité de principe des êtres parlants »" ${ }^{13}$, absolue et inconditionnelle, voilà ce qu'il s'agit de vérifier dans la politique (qu'elle soit " de l'esthétique " ou non), tandis que de l'autre côté, l'ordre dominant s'emploie à séparer les individus en fonction de leurs compétences, afin que se perpétue la légitimité du commandement des uns par les autres.

Dans ses ouvrages strictement philosophiques, l'auteur s'emploie alors à traquer quelques-uns des penseurs critiques qui, à ses yeux, souscrivent au postulat de l'inégalité des intelligences : Althusser dans La Leçon d'Althusser, Marx et Bourdieu dans Le Philosophe et ses pauvres, Platon dans La Mésentente. Mais le terrain privilégié de sa lutte pour l'égalité sera celui de l'art. D'une égalité conçue comme principe, comme hypothèse, voire comme maxime morale ${ }^{14}$, on passe alors à une égalité effectivement réalisée : la présupposition égalitaire n'existe plus seulement dans l'esprit d'un penseur ou d'un péda-

10 Jacques Rancière, Le Spectateur émancipé, Paris, La Fabrique, 2008, p. 29.

11 Ibid., p. 71.

12 Idem.

13 Jacques Rancière, Le Maître ignorant (1987), Paris, Fayard, 2004, p. 67.

14 Ibid., p. 124. 
gogue, mais aussi entre les lignes de la page, dans les plis du corps théâtral ou sur la surface lumineuse de l'écran de cinéma.

La question de l'intention est alors définitivement écartée, comme en témoigne l'exemple de Flaubert, sur lequel le philosophe ne cesse de revenir. Flaubert, en tant qu'individu, fut un réactionnaire notoire, mais cela n'a pas empêché ses romans d'être qualifiés de démocrates par ses contemporains - à raison, d'après Rancière, du moins sur le plan esthétique : Madame Bovary, c'est " la démocratie en littérature " ${ }^{15}$, formule dans laquelle le "en " signale une dépolitisation du concept de démocratie, réinterprété à l'aune de l'écriture et du style. Flaubert, "ce champion supposé de l'art pour l'art $~^{16}$ et de l'écriture aristocratique, se serait en réalité efforcé de renoncer à toute supériorité de l'art sur la vie, d'intégrer à sa prose un certain nombre de temps faibles, de moments insignifiants et d'événements humbles pour leur offrir la même dignité que celle que l'on réservait habituellement aux sujets les plus nobles. Son style réalise donc l'égalité inconditionnelle des sujets, des personnages et des événements. Flaubert ne " dit " rien, il ne cherche ni à prendre position ni à instruire son lecteur. "Son refus même de confier à la littérature aucun message est considéré comme un témoignage de l'égalité démocratique. Il est démocrate, disent ses adversaires, par son parti pris de peindre au lieu d'instruire $»^{17}$. En déjouant la hiérarchie implicite des genres, le roman flaubertien impose sa politique d'une manière qui ne doit rien à ce que l'on désigne habituellement sous ce terme, de sorte que les moyens de sa radicalité demeurent spécifiquement littéraires.

Telle est la politique de l'esthétique selon Jacques Rancière : une manière de vérifier l'égalité des individus et des formes d'expérience par les moyens propres de l'art. Il est donc question d'une politique interne au champ littéraire et artistique, qui tire sa valeur du fait d'être coupée de toute intentionnalité idéologique : lorsque l'art se fait explicitement critique, c'est " au prix de se supprimer comme art ", et c'est la raison pour laquelle Rancière propose une autre voie d'accès à la politique, celle d'un " art qui fait de la politique à la condition expresse de ne pas en faire du tout $»^{18}$. Ainsi, même si l'émancipation politique et l'émancipation artistique " renvoient à un même horizon " (celui de l'égalité), elles " ne s'ajustent pas ${ }^{19}$; penser le contraire, ce serait prendre l'œuvre pour ce qu'elle n'est pas, une action ou un discours, et c'est pour sauvegarder la distinction ontologique de ces deux gestes que Rancière a besoin de construire théoriquement la politique de l'esthétique sans s'appuyer sur ce qu'il conceptualisait sous le nom d'esthétique de la politique.

15 Jacques Rancière, Le Partage du sensible, op. cit., p. 16.

16 Jacques Rancière, "Le moment esthétique de l'émancipation sociale ", La Revue des Livres, $\mathrm{n}^{\circ} 7,2012$, p. 45.

17 Jacques Rancière, Le Partage du sensible, op. cit., p. 16-17.

18 Jacques Rancière, Malaise dans l'esthétique, op. cit., p. 66.

19 Jacques Rancière, La Méthode de l'égalité, Montrouge, Bayard, 2012, p. 103. 


\section{Les réciproques illusoires}

Il existe une profonde asymétrie, au sein de la pensée ranciérienne, entre l'esthétique de la politique et la politique de l'esthétique. Les deux notions ne sont pas seulement indépendantes l'une de l'autre, elles sont également exclusives : pour devenir esthétiquement démocratique, l'œuvre d'art doit renoncer à s'appuyer sur les forces vives d'une théorie ou d'un engagement qui lui préexisterait.

Au fond, l'esthétique de Rancière repose sur deux objectifs opposés : d'une part, fonder en droit une lecture politique des œuvres d'art dans lesquelles ces dernières se verraient attribuer un objectif qui est aussi celui de l'action politique, à savoir l'égalité ; d'autre part, interdire à l'art de confondre ses moyens avec ceux de l'action politique, comme si l'égalité visée par l'un et par l'autre ne devait en aucun cas se matérialiser sous la même forme. Il n'y a qu'un seul concept d'égalité chez Rancière, et pourtant il existe deux manières distinctes d'y parvenir.

Comment expliquer ce dédoublement ? Selon Nicolas Vieillescazes, il faut y voir une prise de position plus générale contre la pensée critique. Sans pour autant rejoindre les rangs du capital et de la domination, Rancière semble en effet se battre contre une " caricature de marxisme " qu'il aurait lui-même construite au fil de ses textes pour s'y opposer ${ }^{20}$. L'idée que l'art puisse être " un instrument au service d'une cause ${ }^{21}$ est plus grave encore, aux yeux de Rancière, que celle qui consiste à priver l'art de toute répercussion politique ou militante. Voilà peut-être pourquoi, plutôt que d'autoriser l'art à intégrer le champ de l'action politique, l'esthétique ranciérienne travaille à le confiner dans son univers propre - quitte, dans un second temps, à redéfinir les lois de cet univers pour qualifier de " politiques » des opérations littéraires, poétiques ou cinématographiques dont le fonctionnement rappelle la structure de l'égalitarisme démocratique.

Que l'on souscrive ou non à cette critique, un problème demeure : malgré l'apparente réversibilité des formules, les termes qui les composent n’ont pas le même sens. C'est le cas, en premier lieu, de la " politique ». Parler d'esthétique de la politique, c'est traiter cette dernière comme un objet d'étude, en partie donné par la situation sociale, intellectuelle, etc., et en partie constitué par le regard de l'observateur. Lorsque Rancière décrit la rencontre de la philosophie platonicienne et de l'hypothèse démocratique dans l'Athènes du Ve siècle avant J.-C., cela lui permet de jeter un nouvel éclairage sur la manière dont s'organise la société grecque, dont elle assigne une place et une fonction à chacun, ou au

20 Nicolas Vieillescazes, «L'esthétique politique de Jacques Rancière : dissolution de la politique dans l'esthétique ? ", La Revue des Livres, n 8, 2012, p. 52.

21 Jacques Rancière, "Le moment esthétique de l'émancipation sociale ", op. cit., p. 46. 
contraire dont un régime gouvernemental spécifique (la démocratie) dérange cette répartition des rôles. Ici, la politique ne désigne pas seulement la mise en œuvre de l'égalité de chacun avec tous, mais la confrontation entre ce processus égalitaire et le partage du sensible sur lequel repose la cité. Le mot " politique " se réfère donc à une situation globale (une société), localisée (dans un espace et un temps donnés) et problématisée (abordée selon une question bien précise, en l'occurrence celle de la visibilité ou de l'audibilité du peuple).

C'est tout le contraire qui se produit dans ses travaux sur la politique de l'esthétique : cette fois, l'idée de "politique » est prise dans un sens élargi, qui matérialise seulement l'application à une discipline ou à un champ d'activité d'une stratégie d'affirmation, "selon un plan élaboré d'avance ${ }^{22}$, sans que cela implique directement l'action collective, le débat public ou les conflits sociaux. Reste seulement l'idée d'un plan conduit en interne par une administration, une personne morale ou un champ d'activité - en l'occurrence le monde de l'art, qui développe ses propres politiques en réponse au système des genres et à la hiérarchie des publics, tous deux en vigueur depuis Aristote ${ }^{23}$. Il reste une forme d'opposition à un ordre majoritaire, mais la dimension critique et stratégique s'estompe indéniablement. De même, les œuvres d'art peuvent bien reposer sur un principe "démocratique ", cela n'en restera pas moins une démocratie "moléculaire " ${ }^{24}$, sans conséquence sur le corps social.

La signification du mot " esthétique " est également différente selon que l'on s'intéresse à la philosophie politique de Rancière ou à sa philosophie de l'art. Dans la seconde, le terme renvoie à une théorie générale qui fait de l'histoire de l'art le terrain d'une lutte entre deux systèmes de pensée inconciliables : d'un côté, le régime représentatif, basé sur la mimesis aristotélicienne, qui attribue à chaque genre une forme et un public privilégiés, ce qui revient à " align[er] les hiérarchies de l'art sur les hiérarchies sociales "; de l'autre, le régime esthétique, reposant sur "l'affirmation qu'il n'y a pas de sujets nobles ou bas, que tout est sujet de l'art ", et donc sur une dé-hiérarchisation généralisée des modes d'expérience ${ }^{25}$. Or, ce processus de redéfinition de l'art est à lui seul une révolution, puisqu'il contribue à imposer dans le champ de l'art l'égalité en droit des objets, des genres, des personnages et des spectateurs à l'égard des lois poétiques. Le désordre qui en découle est d'emblée une blessure infligée à la domination. Mieux : la révolution que constitue l'apparition du régime esthétique est la seule voie de politisation possible pour l'art. Ainsi, dans la première section de Malaise dans l'esthétique ${ }^{26}$, Rancière commence par

22 André Lalande (éd.), Vocabulaire technique et critique de la philosophie (1926), Paris, Presses Universitaires de France, 2002, p. 786.

23 Jacques Rancière, Le Partage du sensible, op. cit., p. 23.

24 Jacques Rancière, Politique de la littérature, Paris, Galilée, 2007, p. 36.

25 Jacques Rancière, Le Destin des images, Paris, La Fabrique, 2003, p. 120.

26 Jacques Rancière, Malaise dans l'esthétique, op. cit., p. 29-84. 
examiner d'autres systèmes de pensée, de Lyotard à Bourriaud, qui sont autant de "politiques de l'esthétique " (c'est-à-dire d'hypothèses de raccordement de la politique et de l'art) auxquelles il s'oppose, et auxquelles il substituera une modalité unique de réalisation du potentiel émancipateur de l'art.

En somme, dans l'expression "politique de l'esthétique », il faut entendre le second terme comme un régime d'identification de l'art et de codification de ses rapports avec l'expérience quotidienne. L'esthétique ne désigne plus une discipline, mais un programme : la rupture historique et philosophique dont elle est le nom est à elle seule l'objet qu'il s'agit de promouvoir. "Le programme "esthétique" est alors proprement celui d'une métapolitique, qui se propose d'effectuer en vérité et dans l'ordre sensible une tâche que la politique ne pourra jamais accomplir que dans l'ordre de l'apparence et de la forme $~_{27}$. Ici, Rancière opère une scission entre les deux versants dont l'union définit traditionnellement la discipline esthétique : la sphère de l'apparence (la politique), et la sphère de la sensation (l'art).

L'écart est total par rapport à son travail sur le partage du sensible. Le terme " esthétique " désignait alors une manière d'envisager le monde qui, précisément, dépassait le cadre de l'art, en imposant l'idée selon laquelle les choses de la pensée ou de la politique sont toujours en même temps des figures, des fictions, des propositions d'agencement du sensible. Ce n'est pas le Rancière flaubertien qui écrit, dans La Mésentente, que «la politique est chose esthétique, affaire d'apparence $»^{28}$, mais bien le Rancière arendtien voire kantien, celui pour qui " esthétique " désigne l'ensemble des événements qui nécessitent d'être appréhendés par les organes sensoriels. Ce Rancière-ci, à la différence de l'autre, s’applique à traiter des œuvres d'art dans le cadre d'une " esthétique générale, c'est-à-dire dans une théorie générale de l'unité de la pensée et du sensible " ${ }^{29}$. Son objet comprend la totalité des "événements du sensible, peu importe qu'ils soient "artistiques" ou non $»^{30}$. Qu'il analyse une ouvre, une action ou un discours, l'observateur n'est pas tenu d'adopter un regard différencié.

\section{Esthétique ou poétique(s)?}

Entre cette indifférenciation esthétique et la politique de l'esthétique que Rancière tente de construire dans ses ouvrages sur l'art, l'écart est tangible. L'auteur l'admet volontiers, notamment lorsqu'il est amené à commenter la proximité sémantique entre ses concepts de politique, d'esthétique et de littérature :

27 Ibid., p. 55.

28 Jacques Rancière, La Mésentente, op. cit., p. 109.

29 Jacques Rancière, "L'historicité du cinéma ", in Antoine de Baecque et Christian Delage (éds), De l'histoire au cinéma, Bruxelles, Complexe, 1998, p. 52 (je souligne).

30 Georges Didi-Huberman, L'Cil de l'histoire, vol. 6 : Peuples en larmes, peuples en armes, Paris, Minuit, 2016, p. 416. 
Je prends « esthétique » en deux sens, l'un large, l'autre restreint. Au sens large, je parle d'une "esthétique de la politique ", pour indiquer que la politique est d'abord une bataille sur les données sensibles elles-mêmes. [...] Au sens restreint, «esthétique » désigne pour moi un régime spécifique de l'art, opposé au régime représentatif. ${ }^{31}$

Ses ouvrages les plus récents insistent davantage sur la seconde acception, en s'efforçant de donner une consistance à ce " paradigme esthétique " dont l'apparition peut à elle seule être considérée comme une "révolution ${ }^{32}$. C'est le régime esthétique qui est l'objet du dernier Rancière, non les œuvres singulières, et encore moins les intentions dont elles résultent.

On peut alors nommer ce qui se trouve exclu de son système : la poétique. Pour l'auteur, ce terme renvoie à une situation ancienne, dans laquelle on pouvait distinguer " une manière de faire - une poiesis - et une manière d'être - une aisthesis - qui est affectée par elle " ${ }^{33}$. Or, dans le régime esthétique de l'art, il n'existe plus de rapport déterminé entre une forme et une signification, ou entre une œuvre et un type de public. La réception (l'aisthesis) est indifférente à la production (la poiesis). Ainsi, l'ouvrage dans lequel Rancière prend ses distances avec l'analyse d'œuvres traditionnelle s'intitule précisément Aisthesis, en réaction à la Mimésis d'Auerbach, dont il conteste localement la lecture du Rouge et le noir ${ }^{34}$, mais peut-être aussi en réaction à la Poétique d'Aristote. Le titre est en lui-même une prise de position : le lecteur ne devra pas s'attendre à ce que l'analyse produise de la signification, car cela nécessiterait encore que quelqu'un (l'artiste, l'œuvre, le critique) en soit défini comme le dépositaire privilégié. L'égalité des affects et des sensations se suffit à elle-même.

Se dessine alors le paradoxe d'une pratique de l'analyse qui ne doit plus rien au faire, au sens, à l'intentionnalité. Le chapitre consacré à Dziga Vertov l'illustre bien : là où la plupart des commentateurs voient un cinéma oscillant entre le formalisme et la propagande, Rancière congédie aussi bien l'un que l'autre. La politique de L'Homme à la caméra, écrit-il, ne se laisse décrire ni dans les termes de la modernisation soviétique, ni dans ceux de l'autonomie formelle revendiquée par les avant-gardes. Ce qui importe, c'est uniquement l'égalité des affects nés de la projection. Pour la désigner, Rancière détourne une nouvelle fois la terminologie révolutionnaire : Vertov n'est pas un cinéaste communiste, il est le créateur d'un " communisme cinématographique " défini comme "équivalence généralisée et accélérée de tous les mouvements " ${ }^{35}$. Une efficacité garantie par le régime esthétique en tant que programme, et par le montage en tant qu'idée de l'art. Les formes précises choisies par Vertov dans son film, elles, n'ont que peu de poids face à cette double détermination.

31 Jacques Rancière, "Littérature, politique, esthétique ", op. cit., p. 159.

32 Jacques Rancière, Aisthesis : Scènes du régime esthétique de l'art, Paris, Galilée, 2011, p. 15.

33 Jacques Rancière, Malaise dans l'esthétique, op. cit., p. 16.

34 Jacques Rancière, Aisthesis, op. cit., p. 63-65.

35 Ibid., p. 284. 
Ainsi s'accomplit le rejet de la poétique, à la fois en tant qu'objet d'analyse (le moment du faire) et en tant que programme théorique (la possibilité pour l'œuvre de produire une signification singulière). C'est ce qui se produit, du moins, si l'on s'en tient à la thèse de la politique de l'esthétique.

Or, il me semble que l'on peut dépasser ce blocage en faisant appel à un autre versant de la pensée ranciérienne : celui, précisément, qui se développe sous le nom d'" esthétique de la politique ». Dans le sillage de la notion de partage du sensible, en effet, le philosophe traitait l'action politique elle-même comme une poétique, c'est-à-dire comme une tentative pour agencer autrement la matière, dans un espace et un temps donnés, avec l'espoir de produire un certain effet (dans l'idéal, une contestation de la logique dominante). Ainsi entendue, la politique a des affinités avec l'art, dans la mesure où elle aspire à faire voir du nouveau au sein d'un ordre traversé par des conflits. La notion de poétique, chez lui, se prête à ce type d'usage ${ }^{36}$. Il devient alors possible de dépasser les limites formulées précédemment, et de jouer la pensée politique de Rancière contre sa philosophie de l'art.

Reprenons le cas de Vertov. Les mouvements produits par ses images ne sont pas seulement horizontalement équivalents, ils sont aussi verticalement pertinents. Ils s'adossent à une esthétique qui leur préexiste : celle de la société soviétique de la fin des années 1920, qui valorise la multiplication des vitesses et des possibilités d'interaction. Il convient donc de prendre en compte l'" horizon d'attente cinétique ${ }^{37}$, nécessairement contextuel, au contact duquel se construisent les formes filmiques - on pourrait aller jusqu'à parler, en paraphrasant Rancière, de partage de la mobilité. De ce point de vue, l'analyse se présente comme une pratique visant à décrire l'entrelacement de deux poétiques : celle de la société soviétique, et celle de Vertov; celle de la politique, dont les lois et les actions participent à donner au monde sensible son apparence définitive, et celle de l'art, qui s'introduit localement dans ce partage pour en déplacer les lignes. Il n'existe qu'un seul univers sensible, qu'une seule "esthétique de la politique ": ce qui varie, ce sont les poétiques du savoir, du pouvoir, du droit, de l'action collective, du cinéma et des arts ; des poétiques qui prennent corps sur l'esthétique de la politique pour la modifier, la fissurer, ou au contraire pour la reconduire.

Dans le système de Rancière, la multiplicité de ces poétiques est éclipsée par l'unicité de la politique de l'esthétique, celle-ci supposant nécessairement une reconduite à l'identique du programme égalitaire flaubertien. À mon sens, il s'agit toutefois d'un refoulé plutôt que d'un impensé. C'est pourquoi j’ai tenté

36 Jacques Rancière, "Histoire des mots, mots de l'histoire ", Communications, vol. 58, n 1 , 1994, p. 89.

37 Ludovic Cortade, Le Cinéma de l'immobilité : Style, politique, réception, Paris, Publications de la Sorbonne, 2008, p. 169-176. 
de montrer qu'il était possible de dépasser cette limite théorique sans quitter le territoire de la pensée ranciérienne - en acceptant simplement d'en jouer les composantes les unes contre les autres. Plutôt qu'une contradiction philosophique interne, il faut voir ici le signe d'une pensée dynamique et exigeante, qui demande à être lue avec rigueur, mais également avec partialité. Pour qu'il existe des lecteurs émancipés, il n'est pas nécessaire que le maitre soit destitué de son pouvoir ; il suffit que ses revendications ne soient pas suivies à la lettre.

\author{
Raphaël Jaudon \\ EA 4160 Passages XX-XXI \\ Université Lyon 2 \\ raphael.jaudon@univ-lyon2.fr
}

\title{
Résumé
}

Cet article aimerait s'interroger sur la division établie par Jacques Rancière entre l'« esthétique de la politique » et la " politique de l'esthétique ». Deux processus que le philosophe tient à maintenir distincts, et qui font exister un écart entre sa philosophie politique et sa philosophie de l'art. Je montrerai en particulier que les termes "politique " et " esthétique " n'ont pas le même sens selon l'expression dans laquelle ils sont utilisés, de sorte que, malgré l'apparente réversibilité des formules, "esthétique de la politique " et " politique de l'esthétique " désignent des projets philosophiquement et idéologiquement opposés.

\section{Mots-clés}

Jacques Rancière, philosophie politique, esthétique et politique, égalité, démocratie littéraire, poétique.

\begin{abstract}
This paper aims to examine the division, in the philosophy of Jacques Rancière, between the "aesthetics of politics" and the "politics of aesthetics". The two processes are kept distinct, which results in the formation of a gap separating Rancière's political philosophy from his philosophy of art. I intend to show that the meanings of "desthetics" and "politics" both vary, depending of the context in which the terms are used. Thus the expressions are not as reversible as they might seem. One may even say that "aesthetics of politics" and "politics of aesthetics" name contradictory purposes, in terms of philosophy and ideology.
\end{abstract}

\section{Keywords}

Jacques Rancière, political philosophy, aesthetics and politics, equality, literary democracy, poetics. 